\title{
DNA barcoding does not separate South American Triatoma (Hemiptera: Reduviidae), Chagas Disease vectors
}

\author{
Silvia Andrade Justi ${ }^{1,2}$, Carolina Dale ${ }^{2}$ and Cleber Galvão ${ }^{2^{*}}$
}

\begin{abstract}
Background: DNA barcoding assumes that a biological entity is completely separated from its closest relatives by a barcoding gap, which means that intraspecific genetic distance (from COI sequences) should never be greater than interspecific distances. We investigated the applicability of this strategy in identifying species of the genus Triatoma from South America.
\end{abstract}

Findings: We calculated intra and interspecific Kimura-2-parameter distances between species from the infestans, matogrossensis, sordida and rubrovaria subcomplexes. In every subcomplex examined we observed at least one intraspecific distance greater than interspecific distances.

Conclusions: Although DNA barcoding is a straightforward approach, it was not applicable for identifying Southern American Triatoma species, which may have diverged recently. Thus, caution should be taken in identifying vector species using this approach, especially in groups where accurate identification of taxa is fundamentally linked to public health issues.

Keywords: Triatominae, Chagas disease, DNA barcoding, Molecular identification

\section{Findings}

DNA barcoding, as proposed by Hebert et al. [1] assumes that a biological entity is completely separated from its closest relatives by a barcoding gap [2], which means that intraspecific genetic distances (from COI sequences) are never greater than interspecific distances.

Triatoma Laporte (Hemiptera: Reduviidae) is the most diverse genus of Chagas Disease vectors, and accurate identification of species is imperative for the efficiency of vector control programs. The Triatoma genus is divided into species complexes and subcomplexes according to geographic distribution and morphological similarity [3].

Recently, Justi et al. [4] reported that the relationships between species assigned to South American Triatomasubcomplexes could not be untangled with the data in hand. We were then prompted to investigate whether DNA barcoding would be a useful tool for identifying

\footnotetext{
* Correspondence: galvao@ioc.fiocruz.br

${ }^{2}$ Laboratório Nacional e Internacional de Referência em Taxonomia de Triatomíneos, InstitutoOswaldo Cruz, FIOCRUZ, Rio de Janeiro, Brazil Full list of author information is available at the end of the article
}

the species within the infestans, matogrossensis, sordida and rubrovaria subcomplexes [3].

Kimura-2-parameter genetic distances [5] were calculated pairwise within each of the above mentionedsubcomplexes (Table 1) using the software MEGA v. 5 [6], and intra and interspecific distances were compared.

In all subcomplexes we observed at least one intraspecific distance greater than interspecific distances (Table 1). To be considered appropriate to identify species within a group, intraspecific distances must always be greater than interspecific ones [2], and therefore DNA barcoding is not accurate for the species-level identification of South American Triatoma. Moreover, the method fails to account for hybridization events, which are naturally observed in Triatoma $[7,8]$, and introgression, which is frequent in nuclear DNA [9]. These considerations argue that Hebert et al.'s [1] proposal of cataloguing biodiversity based only on DNA barcoding may severely underestimate it.

Besides that, as highlighted by Dujardin et al. [10], the morphological changes observed in closely related "species", or "lineages" as we prefer to call them, may have 
Table 1 K2p-distances between species of the Triatoma subcomplexes studied

\begin{tabular}{|c|c|c|c|c|c|c|c|c|c|c|c|c|c|c|c|}
\hline Subcomplex & GenBank & Number & Geographic Origin & & & & & & & & & & & & \\
\hline \multirow[t]{7}{*}{ infestans } & & & & & 1 & 2 & 3 & 4 & 5 & & & & & & \\
\hline & KC249330 & 1 & Chaco Tita, Cochabamba, Bolivia & T. delpontei 53 & & & & & & & & & & & \\
\hline & KC249346 & 2 & Chaco Tita, Cochabamba, Bolivia & T. infestans 44 & 0.021 & & & & & & & & & & \\
\hline & KC249349 & 3 & Cotapachi, Cochabamba, Bolivia & T. infestans 58 & 0.025 & 0.018 & & & & & & & & & \\
\hline & KC249352 & 4 & Mataral, Cochabamba, Bolivia & T. infestans 60 & 0.025 & 0.018 & 0.005 & & & & & & & & \\
\hline & KC249354 & 5 & Ilicuni, Cochabamba, Bolivia & T. infestans 63 & 0.021 & 0.016 & 0.000 & 0.006 & & & & & & & \\
\hline & KC249355 & 6 & Montevideo, Uruguai & T. infestans 69 & 0.072 & 0.061 & 0.064 & 0.069 & 0.103 & & & & & & \\
\hline \multirow[t]{8}{*}{ matogrossensis } & & & & & 7 & 8 & 9 & 10 & 11 & 12 & & & & & \\
\hline & KC249327,KC249328 & 7 & Posse, GO, Brazil & T. costalimai 35 & & & & & & & & & & & \\
\hline & KC249329 & 8 & Chiquitania, Cochabamba, Bolivia & T. costalimai 42 & 0.154 & & & & & & & & & & \\
\hline & KC249360 & 9 & São Gabriel D'oeste, MS, Brazil & T. matogrossensis 192 & 0.134 & 0.138 & & & & & & & & & \\
\hline & KC249361 & 10 & Bahia, Brazil & T. matogrossensis 31 & 0.151 & 0.152 & 0.047 & & & & & & & & \\
\hline & KC249391 & 11 & Pantanal, MT, Brazil & T. vandae 28 & 0.156 & 0.151 & 0.047 & 0.040 & & & & & & & \\
\hline & KC249392 & 12 & Rio Verde do MatoGrosso, MT, Brazil & T. vandae 73 & 0.138 & 0.146 & 0.005 & 0.046 & 0.045 & & & & & & \\
\hline & KC249393,KC249394 & 13 & Rondonópolis, MT, Brazil & T. vandae 74 & 0.158 & 0.150 & 0.048 & 0.059 & 0.007 & 0.052 & & & & & \\
\hline \multirow[t]{13}{*}{ rubrovaria } & & & & & 14 & 15 & 16 & 17 & 18 & 19 & 20 & 21 & 22 & 23 & 24 \\
\hline & KC249322 & 14 & São Gerônimo, RS, Brazil & T. carcavalloi 78 & & & & & & & & & & & \\
\hline & KC249323 & 15 & Caçapava do Sul, RS, Brazil & T. circummaculata 120 & 0.039 & & & & & & & & & & \\
\hline & KC249324 & 16 & Sítio Faxina, Piratini, RS, Brazil & T. circummaculata 121 & 0.029 & 0.025 & & & & & & & & & \\
\hline & KC249325 & 17 & Sítio Faxina, Piratini, RS, Brazil & T. circummaculata 122 & 0.017 & 0.039 & 0.033 & & & & & & & & \\
\hline & KC249356 & 18 & Nova Petrópolis, RS, Brazil & T. klugi 75 & 0.018 & 0.037 & 0.031 & 0.017 & & & & & & & \\
\hline & KC249369 & 19 & Sítio Faxina, Piratini, RS, Brazil & T.rubrovaria 123 & 0.055 & 0.023 & 0.029 & 0.055 & 0.057 & & & & & & \\
\hline & KC249370 & 20 & Sítio venda da Lagoa, Canguçu, RS, Brazil & T.rubrovaria 134 & 0.065 & 0.052 & 0.065 & 0.065 & 0.061 & 0.070 & & & & & \\
\hline & KC249372 & 21 & SítioFaxina, Pinheiro Machado, RS, Brazil & T.rubrovaria 136 & 0.042 & 0.019 & 0.027 & 0.036 & 0.036 & 0.031 & 0.035 & & & & \\
\hline & KC249373 & 22 & Sítiovenda da Lagoa, Canguçu, RS, Brazil & T.rubrovaria 140 & 0.038 & 0.020 & 0.019 & 0.043 & 0.040 & 0.029 & 0.032 & 0.012 & & & \\
\hline & KC249374 & 23 & Canguçu, RS, Brazil & T.rubrovaria 156 & 0.039 & 0.020 & 0.019 & 0.045 & 0.042 & 0.029 & 0.032 & 0.012 & 0.000 & & \\
\hline & KC249375 & 24 & Caçapava do Sul, RS, Brazil & T.rubrovaria 76 & 0.021 & 0.029 & 0.021 & 0.016 & 0.016 & 0.033 & 0.074 & 0.034 & 0.038 & 0.038 & \\
\hline & KC249376 & 25 & Quevedos, RS, Brazil & T.rubrovaria 77 & 0.029 & 0.030 & 0.043 & 0.022 & 0.029 & 0.046 & 0.065 & 0.031 & 0.046 & 0.048 & 0.026 \\
\hline \multirow[t]{4}{*}{ sordida } & & & & & 26 & 27 & 28 & 29 & 30 & 31 & 32 & 33 & 34 & & \\
\hline & KC249338 & 26 & Rivadaria, Argentina & T. garciabesi 89 & & & & & & & & & & & \\
\hline & KC249342 & 27 & Santa Cruz, Bolívia & T. guasayana 55 & 0.077 & & & & & & & & & & \\
\hline & KC249343 & 28 & Santa Cruz, Bolívia & T. guasayana 82 & 0.065 & 0.056 & & & & & & & & & \\
\hline
\end{tabular}


Table 1 K2p-distances between species of the Triatoma subcomplexes studied (Continued)

\begin{tabular}{|c|c|c|c|c|c|c|c|c|c|c|c|c|}
\hline KC249379,KC249380 & 29 & Romerillo, Cochabamba, Bolivia & T. sordida 46 & 0.029 & 0.060 & 0.060 & & & & & & \\
\hline KC249381,KC249382 & 30 & Romerillo, Cochabamba, Bolivia & T. sordida 47 & 0.030 & 0.061 & 0.061 & 0.000 & & & & & \\
\hline KC249383 & 31 & La Paz, Bolívia & T. sordida 83 & 0.081 & 0.013 & 0.063 & 0.066 & 0.066 & & & & \\
\hline KC249384 & 32 & Pantanal, MS, Brazil & T. sordida 85 & 0.069 & 0.012 & 0.062 & 0.065 & 0.065 & 0.025 & & & \\
\hline KC249385 & 33 & Santa Cruz, Bolívia & T. sordida 86 & 0.043 & 0.082 & 0.074 & 0.035 & 0.035 & 0.073 & 0.082 & & \\
\hline KC249387 & 34 & San Miguel Corrientes, Argentina & T. sordida 88 & 0.061 & 0.058 & 0.063 & 0.070 & 0.071 & 0.058 & 0.055 & 0.052 & \\
\hline KC249388 & 35 & Poconé, MT, Brazil & T. sordida 90 & 0.069 & 0.017 & 0.058 & 0.075 & 0.075 & 0.031 & 0.011 & 0.078 & 0.051 \\
\hline
\end{tabular}

Highlighted distances deviate from the DNA barcoding premis that intraspecific distances are smaller than interspecific distances. 
led taxonomists to rush into describing subspecies or species, even genera. Molecular phylogenetic studies are in their infancy in unravelling the evolution of Triatominae, and a comprehensive molecular phylogeny, including more than one specimen for most lineages, was published only in 2014 [4], although several analyses were conducted focusing on small species groups. Taken together, these statements make it clear that further investigations of Triatominae evolution are long overdue, preferably integrating morphological, molecular and ecological data.

Lineage evolution has not occurred, but it is happening now. Concerning lineages designated in the infestans complex (including the subcomplexes studied here), separation is much clearer in terms of morphology than in molecular systematics. In cases where lineages have not reached reciprocal monophyly, defining taxonomic entities is not a straightforward issue [11]. Therefore caution is necessary, especially in a group where accurate identification of taxa is fundamentally linked to public health issues.

\section{Conclusions}

Although DNA barcoding is a straightforward approach, it was not applicable for identifying Southern American Triatoma species, which may have diverged recently. Thus, caution should be taken in identifying vector species using this approach, especially in groups where accurate identification of taxa is fundamentally linked to public health issues.

\section{Competing interests}

The authors declare that they have no competing interests.

\section{Authors' contributions}

SAJ designed the study, acquired data, performed all the analyses, interpreted the results, drafted and reviewed the manuscript. CD designed the study, acquired data, performed all the analyses, interpreted the results, drafted and reviewed the manuscript. CG designed the study and reviewed the manuscript. All authors read and approved the final version of the manuscript.

\section{Authors' information}

CD is PhD student funded by Instituto Oswaldo Cruz and SAJ is a Post Doctoral Fellow funded by CNPq.

\section{Acknowledgements}

We thank Carlos G. Schrago for reviewing our manuscript and for the usefu comments.

\section{Author details \\ 'Universidade Federal do Rio de Janeiro, Instituto de Biologia, Laboratório de Biologia Evolutiva Teórica e Aplicada, Rio de Janeiro, Brazil. '²aboratório Nacional e Internacional de Referência em Taxonomia de Triatomíneos, InstitutoOswaldo Cruz, FIOCRUZ, Rio de Janeiro, Brazil.}

Received: 13 September 2014 Accepted: 4 November 2014 Published online: 21 November 2014

\section{References}

1. Hebert PDN, Cywinska A, Ball SL, DeWaard JR: Biological identifications through DNA barcodes. Biol Sci: In Proc Roy SocLond B; 2003:313-321.
2. Meyer CP, Paulay G: DNA barcoding: error rates based on comprehensive sampling. PLoS Biol 2005, 3(Suppl. 12):e422.

3. Schofield CJ, Galvão C: Classification, evolution, and species groups within the Triatominae. Acta Trop 2009, 110(Suppl 2-3):88-100.

4. Justi SA, Russo AMC, Santos-Mallet JR, Obara MT, Galvão C: Molecular phylogeny of Triatomini (Hemiptera: Reduviidae: Triatominae). Parasit Vectors 2014, 7:149.

5. Kimura M: A simple method for estimating evolutionary rates of base substitutions through comparative studies of nucleotide sequences. J Mol Evol 1980, 16:111-120.

6. Tamura K, Peterson D, Peterson N, Stecher G, Nei M, Kumar S: MEGA5: molecular evolutionary genetics analysis using maximum likelihood, evolutionary distance, and maximum parsimony method. Mol Biol Evol 2011, 28:2731-2739.

7. Carcavallo RU, Girón IG, Jurberg J, Galvao C, Noireau F, Canale D: Mutations, Hybrids and Teratologies. In Atlas of Chagas Disease Vectors in the Americas. Edited by Editora FIOCRUZ. Rio de Janeiro: Carcavallo, Girón, Jurberg, Lent; 1998:515-536.

8. Costa J, Peterson AT, Dujardin JP: Morphological evidence suggests homoploid hybridization as a possible mode of speciation in the Triatominae (Hemiptera, Heteroptera, Reduviidae). Inf Gen Evol 2009, 9:263-270.

9. Zhang $A B$, Sota $T$ : Nuclear gene sequences resolve species phylogeny and mithocondrial introgression inLeptocarabusbeetles showing trans-species polymorphisms. Mol Phylogenet Evol 2007, 45:534-546.

10. Dujardin JP, Costa J, Bustamante D, Jaramillo N, Catalá S: Deciphering morphology in Triatominae: the evolutionary signals. Acta Trop 2009, 110(Suppl 2-3):101-111.

11. Arnold ML: Natural Hybridization and species concepts. In Natural Hybridization and Evolution. Oxford: Arnold: Oxford University Press; 1997:11-22.

doi:10.1186/s13071-014-0519-1

Cite this article as: Justi et al:: DNA barcoding does not separate South American Triatoma (Hemiptera: Reduviidae), Chagas Disease vectors. Parasites \& Vectors 2014 7:519.

\section{Submit your next manuscript to BioMed Central and take full advantage of:}

- Convenient online submission

- Thorough peer review

- No space constraints or color figure charges

- Immediate publication on acceptance

- Inclusion in PubMed, CAS, Scopus and Google Scholar

- Research which is freely available for redistribution 